\title{
Diazentrieren - ein einfaches Verfahren zur Ultrafiltration in Zentrifugen
}

\author{
Von H. HOFFMEISTER und W. TARNOWSKI
}

\author{
Aus der 1. Medizinischen Universitätsklinik (Direktor: Prof. Dr. H. Bartelbeimer) und dem Pbysiologisch-Cbemischen \\ Institut der Universität Hamburg (Direktor: Prof. Dr. J. Kübnau)
}

(Eingegangen am 24. Mai 1967)

Ein cinfaches Gerät wird beschrieben, das eine schnelle und schonende Enteiweißung von biologischen Flüssigkeiten und Gewebeextrakten durch „Diazentrieren“, d. h. durch Ultrafiltration im Schwerefeld einer Zentrifuge, gestattet. Die Eigenschaften dieses Gerätes, die Möglichkeiter für seine Anwendung sowie seine Vor- und Nachteile werden diskutiert.

A simple apparatus is described for the rapid and protective deproteinisation of biological fluids and tissue extracts by "diacentration“, i. e., by ultra-filtration in the gravitational field of a centrifuge. The apparatus, its applications and its advantages and disadvantages are discussed.

Die Trennung hochmolekularer von niedermolekularen Stoffen ist eine häufige Voraussetzung biochemischer und klinisch-chemischer Arbeiten. Vor allem ist die Enteiweißung von Proben ein Alltagsproblem. Meist werden Serum, Liquor oder Gewebeextrakte durch Zugabe von Säuren enteiweißt. Aber diese Methode, durch die die Raumstruktur der Proteine zerstört wird, ist in mancher Hinsicht unbefriedigend.

Wir haben daher ein einfaches Gerät entwickelt, in dem biologische Flüssigkeiten ohne Säurezusatz enteiweißt werden können. Es besteht im Prinzip aus einer gestützten, semipermeablen Membran, die von allen niedermolekularen Stoffen im Schwerefeld einer Zentrifuge quantitativ passiert wird. Dieses Verfahren nennen wir - da es eine Kombination von Dialyse und Zentrifugation ist - „Diazentrieren“. Das Diazentrat enthält die kleinen Moleküle in derselben Konzentration wie die eiweißhaltige Ausgangslösung.

\section{Beschreibung des Geräts}

Wir haben aufgrund von Versuchsreihen mit perforierten Plastikbechern, bei denen Zahl und Größe der Bohrungen wechselten, einen Prototyp entwickelt, der jetzt auf den Markt kommt ${ }^{1}$ ). Abbildung 1 zeigt eine schematische Zeichnung des Geräts. Es besteht aus einem feinen Drahtkorb in Form eines Zentrifugenbechers, gestützt von Kunststoffleisten. Dieser versteifte Siebteil des Gerätes wird in einen Außenbecher eingesetzt, der das Diazentrat auffängt. In unseren bisherigen Versuchen haben wir die eiweißhaltige Flüssigkeit in handelsübliche Dialysierschläuche (z. B. Kalle AG, Wiesbaden, Nr. 19997) gegeben, diese oberhalb des Inhalts verknotet und in den Siebteil geschoben. Für das Serienfabrikat werden aber oben offene, steife Dialysierhüllen vorbereitet. Der gefüllte Siebteil wird in den Außenbecher eingesetzt und zentrifugiert. Dabei sammelt sich das Diazentrat im Auffangbecher. Seine Menge ist eine Funktion der Zentrifugalbeschleunigung, der Zentrifugationszeit und derFlüssigkeitssäule über der semipermeablen Membran. Die Abhängigkeit der Diazentratmenge von der Menge der Ausgangslösung zeigt Abbildung 2. Die Filtrationsgeschwindigkeit kann dadurch gesteigert werden, daß die Flüssigkeitssäule über der Dialysiermembran durch Uberschichten der Lösung mit einer chemisch inerten, spezifisch leichteren und nicht diazentrierbaren Flüssigkeit (z. B. Paraffin) vergrößert wird.

\section{Beschaffenheit der Diazentrate}

Das Diazentrat ist eiweißfrei. Selbst mit sehr wirksamen Eiweißfällungsmitteln war niemals eine Trübung nachzuweisen. Nur $0,5-1,0 \%$ biuretpositiver Stoffe - wahrscheinlich nicht fällbare, niedermolekulare Peptide - passieren die Membran (Abb. 3). Auch

1) Firma R. Brand, Wertheim/Main, Glashütte.

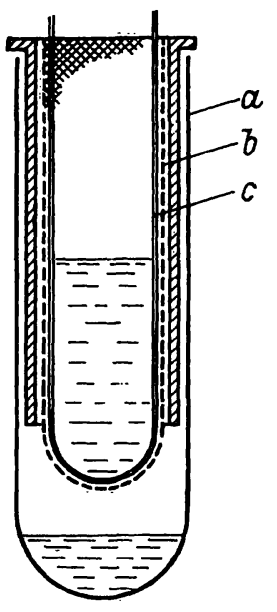

Abb. 1

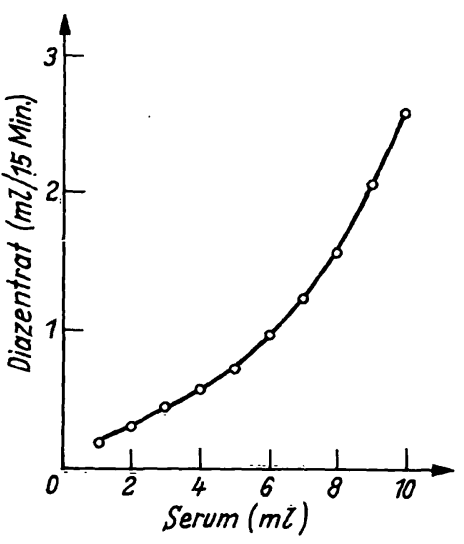

Abb. 2
Abb. 1

Gerät zur Gewinnung von Ultrafiltraten in Zentrifugen. $a=$ Zentrifugenbecher aus Metall oder Porex $b=$ Siebeinsatz aus rostfreiem Stahlgewebe mit Kunststoffverstärkung c = semipermeable Hülse (z. B. Dialysierschläuch)

Abb. 2

Filtrationsleistung in Abhängigkeit von der vorgegebenen Menge menschlichen Serums.

Die Werte wurden nach 15 Min. Zentrifugation bei $20000 \mathrm{~g}$ mit dem in Abbildung 1 dargestellten und im Text beschriebenen Prototyp erhalten

andere hochmolekulare Stoffe, wie Glykogen und Nucleinsäuren, werden quantitativ zurückgehalten. Kleinere Moleküle wie Glucose, Harnstoff, Aminosäuren, treten ohne Konzentrationsänderung ins Ultrafiltrat über. Abbildung 4 zeigt, daß Glucose mindestens bis zu einer Konzentration von $1 \mathrm{~g} / 100 \mathrm{ml}$ aus menschlichem Plasma mit einem Eiweißgehalt von $7,6 \mathrm{~g} / 100 \mathrm{ml}$ quantitativ diazentriert werden kann. Dasselbe gilt für Aminosäuren in Konzentrationen bis zum 10fachen der Norm (Abb. 5). Die Eichkurven für die Aminosäuren der Abbildung 5 wurden mit Ninhydrin bestimmt. Bei der Messung von Alanin mit Glutamat-Pyruvat-Transaminase $\left.(5)^{2}\right)$ fanden wir eine Eichgerade, die gegenüber der Ninhydringeraden der Abbildung 5 geringfügig parallel nach unten verschoben war. Da diese enzymatische Reaktion wegen der Michaeliskonstanten des Enzyms keine Endwertbestimmung ist, glauben wir, daß die Reaktion durch Inhaltsstoffe des Dialyseschlauches gestört wird. - Setzt man dialysiertem menschlichen Plasma ein Gemisch von 20 Aminosäuren zu (Konzentration jeder Aminosäure: $0,1 \mathrm{M}$ ), dann findet man im Diazentrat dieser eiweißhaltigen Standardlösung nach quantitativer Säulenchromatographie in einem Technicon-Autoanalyzer für jede Aminosäure denselben Wert wie in einer wäßrigen Stammlösung gleicher Konzentration.

2) Der Trivialname Glutamat-Pyruvat-Transaminase wird hier gebraucht für das Enzym L-Alanin: 2-Oxoglutarat Aminotrans:ferase (EC 2.6.1.2). 


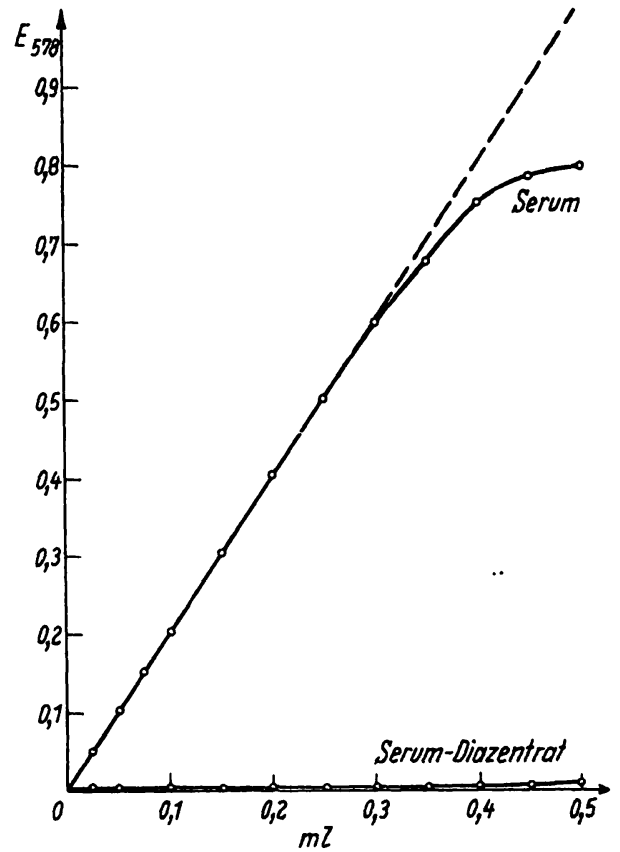

Abb. 3

Eiweißgehalt in steigenden Serummengen und deren Diazentraten (Biuretmethode (1))

Ab $0,5 \mathrm{~m} /$ Filtrat sind sehr kleine Mengen biuretpositiver Stoffe im Diazentrat nachweisbar

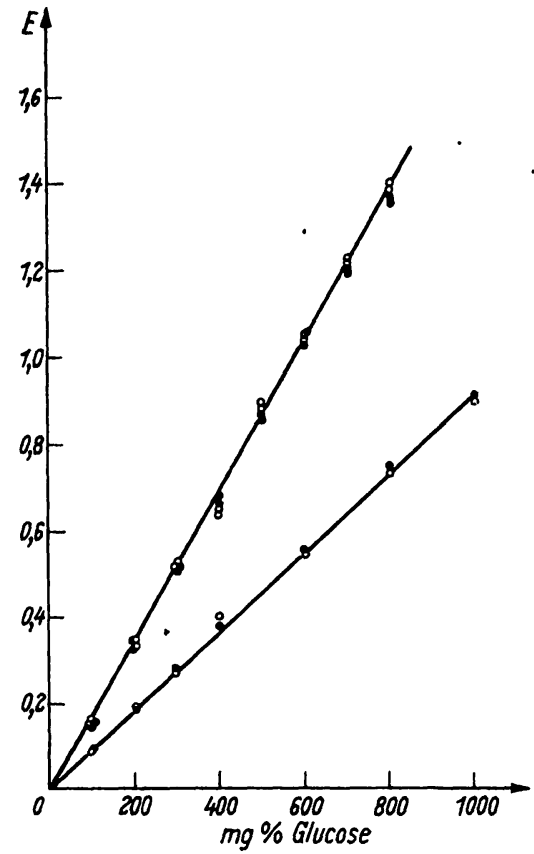

Abb. 4

Glucosegehalt in menschlichen Seren und deren Diazentraten. Nachweis mit Triphenyltetrazoliumchlorid (2) (obere Kurve, $E_{436}$ ) und Hexokinase (3) (untere Kurve, $E_{334}$ )

Menschlichem Serum wurden steigende Mengen Glucose zugesetzt. Die Diazentrate wurden jeweils ohne den Schritt der Eiweißfällung mit Säure verwendet - Serum Diazentrat

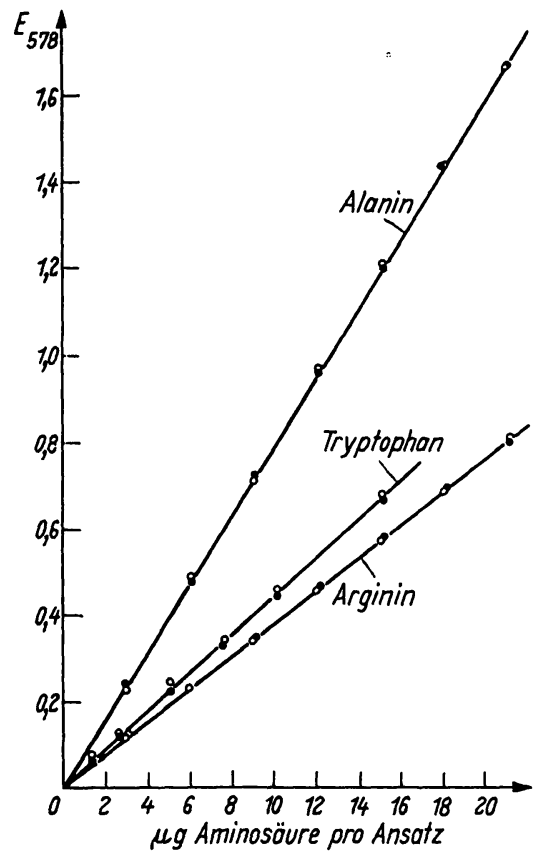

Abb. 5

Alanin-, Tryptophan- und Arginingehalt in menschlichen Seren und deren Diazentraten

Humanplasma wurde bei $2^{\circ}$ erschöpfend gegen 0,9proz. $\mathrm{NaCl}-\mathrm{Lösung}$ dialysiert. Dem dialysierten Plasma wurden steigende Aminosäuremengen zugesetzt. Die Diazentrate wurden mit einer wäßr. Lösung der Aminosäuren gleicher Konzentration verglichen. Bestimmung mit Ninhydrin nach STEIN und MOORE (4) - Serum Diazentrat

\section{Diskussion}

Anlaß für die Entwicklung des beschriebenen Gerätes war die Notwendigkeit, Aminosäuren aus eiweißhaltigen Flüssigkeiten oder Gewebeextrakten für die säulenchromatographische Analyse möglichst einfach, schnell und schonend von Makromolekülen zu trennen. Die oft empfohlenen und angewandten Verfahren, z. B. die Enteiweißung-durch Pikrinsäure mit anschließender Entfernung des Fällungsmittels an Ionenaustauschern (6-9), sind überaus umständlich und - wegen der großen Zahl und der Art der Arbeitsgänge - nicht hinreichend genau. Diesen Methoden gegenüber ist das Diazentrieren von biologischen Flüssigkeiten und Extrakten sehr viel schneller, geñauer und schonender. Binnen 20 Min. kann man $z$. B. aus $5 \mathrm{~m}$ ' Serum in einer Zentrifuge bei etwa $20000 \mathrm{~g}$ ausreichende Diazentratmengen von so vielen Proben erhalten wie der Rotor aufnehmen kann. Unser Verfahren ist auch einfacher als die Gleichgewichtsdialyse gegen große Volumina (7, 10). Gegenüber der Druckfiltration $(7,11)$ hat es den Vorteil, daß - abgesehen von dem geringeren technischen Aufwand - die Filtrationsfläche wesentlich größer ist als die der gebräuchlichen Druckfilter und daher sehr viel später durch aufgelagerte Eiweißmembranen verschlossen wird. - Zudem kann auch dort, wo $z w a r$ eine Kühlzentrifuge, aber kein Kühlraum vorhanden ist, in der Kälte gearbeitet werden.
Der Gedanke, das Schwerefeld einer Zentrifuge für die Druckfiltration einzusetzen, ist nicht neu. Die ersten Geräte (12-15) hatten aber so viele technische Mängel, daß sie sich nicht durchsetzten. Daher konstruierten FeldmanN und Mitarb. (16) 1950 einen gläsernen Diazentrierbecher, der die Fehler seiner Vorläufer vermied. Aber sowohl dieser als auch dessen vereinfachte Modifikation von Toribara (17) gerieten wieder in Vergessenheit, wahrscheinlich, weil die Geräte bei mehr als etwa $1300 \mathrm{~g}$ zerbrachen und man mithin große Probenmengen haben mußte, um relativ kleine Diazentratmengen zu erhalten. Um die Ausbeute zu erhöhen, zog SIEGMUND (18) die Dialyseschläuche vor schärferem Zentrifugieren in genau passende Nylonschläuche ein. Diese Maßnahme aber - zusammen mit den relativ schwer zu reinigenden Bechereinsätzen - hat die Anwendung des Gerätes für Reihen- und Routineuntersuchungen wahrscheinlich verhindert. Auch ein Diazentriereinsatz von METZNER (19) aus mehreren Teilen und mit einem kreisrunden Membraneinsatz hat sich, da er zu kompliziert war, nicht durchgesetzt. Das Gerät StEkElENBURGs (20) hat sich uns nicht bewährt. Sein freihängender Dialyseschlauch ist schwer $\mathrm{zu}$ befestigen und reißt schon bei einer Belastung unter $2000 \mathrm{~g}$. - Alle diese Nachteile hat unser Gerät nicht.

Dưrch Diazentrieren können Schnelligkeit und Genauigkeit derjenigen klinisch-chemischen Routinebe- 
stimmungen gesteigert werden, für die biologisches Material enteiweißt werden muß. Bei quantitativen Bestimmungen erfordert die Enteiweißung mit denaturierenden Substanzen vor der eigentlichen Reaktion das Abmessen eines Probenaliquots und die Zugabe des Fällungsmittels. Diese beiden quantitativen Schritte, die lange dauern und die Genauigkeit der Bestimmungen mindern, entfallen aber, wenn die Proben diazentriert werden. Die Methode könnte daher klinisch-chemische Routinelaborarbeiten fühlbar entlasten.

Auch ist es möglich, durch Diazentrieren den Anteil eines Stoffes zu bestimmen, der im Serum oder Liquor fest an Eiweiß gebunden ist. Untersuchungen dieser Art, die wir an der Harnsäure des menschlichen Serums begonnen haben, zeigen, daß die Eiweißbindungskapazität des Blutes für solche Stoffe offenbar ein pathophysiologisches Kriterium mancher Krankheitssyndrome ist.

\title{
Literatur
}

1. Campbell, W. R. und M. T. Hanna, J. biol. Chemistry 119, 9 (1937). - 2. Lorentz, K. und H. Hoffmeister, Mikrichimica Acta (Wien) 1062 (1966). - 3. Barthelmar, W. und R. Czok, Klin. Wschr. 40, 585 (1962). - 4. Moore, S. und W. H. Stein, J. biol. Chemistry 211, 907 (1954). - 5. Pfleiderer, G. und T. Wieland, Ann. Acad. Sci. fenn. A II, 60, 381 (1955). - 6. Hamilton, P. B. und D. D. van SLYKe, J. biol. Chemistry 150, 231 (1943). 7. Stern, W. H. und S. Moore, J. biol. Chemistry 211, 915 (1954). -8. Tallan, H. H., S. Moore und W. H. Stern, J. biol. Chemistry 219, 257 (1956). - 9. Beckman Amino Acid Analyzer Model 120 B Instruction Manual, Part 7-1 (1962). - 10. Hamilton, P. B. und R. M. Archibald, Indian Eng. Chem. Anal., Ed. 16, 136
(1944). - 11. Prescott, B. A. und H. Waelsch, J. biol. Chemistry 167, 855 (1947). - 12. Ferry, J. D., Chem. Revs. 18, 373 (1936). 13. Nrcholas, H. O., J. biol. Chemistry 97, 475 (1932). - 14. Rehberg, P. B., Acta Physiol. Scand. 5, 305 (1943). - 15. WaArd, D. J. DE, Arch. néerland. physiol. 2, 530 (1918). - 16. FELdMAN, I., R. A. Danley und J. F. O'Leary, Analytic. Chem. 22, 837 (1950). - 17. Toribara, T. Y., Analytic. Chem. 25, 1286 (1953). - 18. SIEGmund, P., Habilitationsschrift, Freie Universität Berlin, 1962. - 19. Metzner, H., Naturwissenschaften 14, 388 (1953). - 20. Stekelenburg, G. J. und J. Desplanque, Technicon Instruments Company Ltd., $4^{\text {th }}$ Amino Acid Colloq., London, p. 83 (1966).

Dozent Dr. W. Tarnowski

2 Hamburg 20, Martinistraße 52

\section{Vergleichende Untersuchungen über die Antivitamin-B -Wirkung von L- und D-Penicillamin}

\author{
Von F. Körber, G. Hasenbank und P. Siegmund \\ Aus dem Pbysiologisch-Chemischen Institut der Freien Universität Berlin (Direktor: Prof. Dr. Dr. E. Schütte)
}

(Eingegangen am 22. Juni 1967)

Applikation von täglich $10 \mathrm{mg}$ L- bzw. D-Penicillamin intraperitoneal über drei Wochen führt bei Ratten

1. nach Belastung mit $40 \mathrm{mg}$ Tryptophan zu einer Xanthurensäure-Ausscheidung von $5,7 \mathrm{mg}$ nach L-Penicillamin, von $0,6 \mathrm{mg}$ nach D-Penicillamin gegenüber $0,2 \mathrm{mg}$ bei den Kontrolltieren.

2. zu einer 20 proz. Verminderung der Aktivität von Aspartataminotransferase in der Leber nach L-Penicillamin, während nach DPenicillamin kein Unterschied zu den Kontrolltieren auftritt.

3. zu einer 50 proz. Verminderung der Aktivität von Alaninaminotransferase in der Leber nach L-Penicillamin und einer 20 proz. Verminderung nach D-Penicillamin.

Rats received $10 \mathrm{mg}$. of $\mathrm{L}$ - or $\mathrm{D}$-penicillamine daily over a period of three weeks.

1. The excretion of xanthurenic acid after loading with $40 \mathrm{mg}$. tryptophan increased from $0.2 \mathrm{mg}$. in the control animals to $5.7 \mathrm{mg}$. after L-penicillamine and $0.6 \mathrm{mg}$. after D-penicillamine.

2. The aspartate-aminotransferase activity in the liver of L-penicillamine-treated animals was decreased by $20 \%$, while $\mathrm{D}$-penicillamine had no effect.

3. The alanine-aminotransferase activity was decreased by $50 \%$ in the liver after treatment with L-penicillamine, and by $20 \%$ after $\mathrm{D}$ penicillamine.

In einer Reihe von Arbeiten haben DU VIGNEAUd und Mitarbeiter (1-5) gezeigt, daß dem Futter zugesetztes L-Penicillamin das Wachstum von Ratten hemmt. Die Tiere zeigten Hautsymptome, wie sie bei Vitamin- $\mathrm{B}_{6}$ Mangel auftreten und schieden nach Tryptophanbelastung große Mengen Xanthurensäure aus. Die Transaminaseaktivitäten im Leberhomogenat waren vermindert. Durch Vitamin- $\mathrm{B}_{6}$-Gaben konnten diese Wirkungen verhindert werden. Auch für D-Penicillamin, das für die Behandlung der Wrusonschen Erkrankung verwendet wird, ist eine Antivitamin- $\mathrm{B}_{6}$-Wirkung be- schrieben worden (6-8). Eine Untersuchung beider Penicillamin-Antipoden unter vergleichbaren Versuchsbedingungen fehlt bisher jedoch.

\section{Material und Methoden \\ Reagenzien und Referenzsubstanzen}

L- und D-Penicillamin erhielten wir von Heyl und Co. ${ }^{1}$ ), Berlin. Die Reinheit wurde durch Messung der optischen Drehung im lichtelektrischen Polarimeter (Zeiss), durch SH-Gruppentitration mit

1) Wir danken der Firma für die kostenlose Uberlassung dieser Substanzen. 